\title{
ORBIT CONTROL AT THE ALS BASED ON SENSITIVITY MATRICES*
}

\author{
H. Nishimura, L. Schachinger, and H. Ohgaki
}

Advanced Light Source

Accelerator and Fusion Research Division

Lawrence Berkeley Laboratory.

University of California

Berkeley, CA 94720

April 1995

Paper presented at the 1995 Particle Accelerator Conference, Dallas,

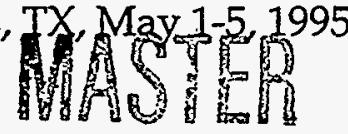

*This work was supported by the Director, Office of Energy Research, Office of Basic Energy Sciences, Materials Sciences Division, of the U.S. Department of Energy, under Contract No. DE-AC03-76SF00098. 


\section{DISCLAIMER}

This report was prepared as an account of work sponsored by an agency of the United States Government. Neither the United States Government nor any agency thereof, nor any of their employees, make any warranty, express or implied, or assumes any legal liability or responsibility for the accuracy, completeness, or usefulness of any information, apparatus, product, or process disclosed, or represents that its use would not infringe privately owned rights. Reference herein to any specific commercial product, process, or service by trade name, trademark, manufacturer, or otherwise does not necessarily constitute or imply its endorsement, recommendation, or favoring by the United States Government or any agency thereof. The views and opinions of authors expressed herein do not necessarily state or reflect those of the United States Government or any agency thereof. 


\section{DISCLAIMER}

Portions of this document may be illegible in electronic image products. Images are produced from the best available original document. 


\title{
ORBIT CONTROL AT THE ALS BASED ON SENSITIVITY MATRICES*
}

\author{
H. Nishimura, L. Schachinger and H. Ohgaki** \\ Lawrence Berkeley Laboratory, University of California, CA94720 USA
}

\section{INTRODUCTION}

A third-generation synchrotron-light source storage ring requires accurate orbit correction because its lattice is very sensitive to magnet imperfections and misalignments. If model-based control is used, calibration of this model is required in advance. Therefore, it is preferable to prepare some kind of model-free orbit control scheme that is complementary to model-based control. A sensitivity matrix (Smatrix) works effectively as the kernel of a model-free orbit control system for a given optics of a lattice. This paper describes recent efforts in this respect at Advanced Light Source (ALS)[1].

\section{SMATRIX}

\section{A. Definition}

An Smatrix, $S=\left(S_{i j}\right)$, represents the effect of each steering magnet on each beam position monitor (BPM) around the ring as $\delta \mathrm{Z}_{\mathrm{i}}=\Sigma \mathrm{S}_{\mathrm{ij}} \delta \mathrm{K}_{\mathrm{j}}$, where $\delta \mathrm{Z}_{\mathrm{i}}$ is a change in the position at the $\mathrm{i}$-th $\mathrm{BPM}$ and $\delta \mathrm{K}_{\mathrm{j}}$ is the diflection given by the $\mathrm{j}$-th steering magnet.

\section{B. Calculation and Measurement}

A direct way of calculating a theoretical Smatrix is to use a program that can calculate either a closed orbit or linear optics. Measuring is also a straightforward method. An automated Smatrix measurement, in the ALS Storage Ring, takes about 40 minutes horizontally and 30 minutes vertically, and a quicker version is presently being tested so we can measure the matrices in both planes in 10 minutes.

\section{Analysis}

An Smatrix contains information, at BPM and Steering Magnet locations, of the linear optics contributions under which it was measured or calculated. Therefore, it is possible to reconstruct the lattice optics from it. This is usually a timeconsuming and complex calculation. During the ALS commissioning period, our first effort was made in collaboration with BESSY[2]. A more complete analysis has been going on in collaboration with SSRL at SLAC[3].

In this paper, we will only discuss a simple analysis to check the validity of a measured Smatrix, by checking the symmetry of the ring they reflect. Let $N$ and $M$ be the number of BPMs and steering magnets per sector, respectively. Then

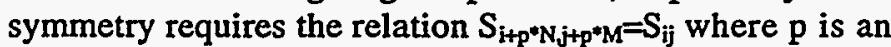
integer. Assume there are errors in the calibration-factor set $\left(c_{i}, d_{j}\right)$ such that $\delta Z_{i}=c_{i} \delta Z_{o i}$ and $\delta K_{j}=d_{j} \delta K_{o j}$, where $\delta Z_{o i}$ and $\delta \mathrm{K}_{\mathrm{oj}}$ are ideal values. Then a least-square fit gives $\left(c_{i}, d_{j}\right)$.

If there is a good symmetry with $c_{i}=d_{j}=1.0$ the Smatrix can be taken as valid. In this case, all the BPMs and steering magnets are working properly during the measurement and the calibration factors are due to device-calibration errors and beta-function beat at the device locations. The beta-beat can be measured by other methods [4] and it is possible to extract the device-calibration errors. In the case of the ALS storage ring, it turned out that the calibration errors of BPMs and steering magnets are negligibly small and all the calibration factors are essentially caused by the beta-beat.

\section{SMATRIX-BASED ORBIT CONTROL}

The measured Smatrices at the ALS storage ring indicate that there is a beta-beat in the order of a few percent around the ring that is relevant for a model-based orbit control. Therefore, we have been working on an Smatrix-based orbit control.

With a fixed lattice configuration, it is possible to use Smatrices to set up parameters for several kinds of orbit correction schemes, including those that use local bumps or the singular value decomposition (SVD) method[5]. Figure 1 shows the components of our Smatrix-based orbit control.

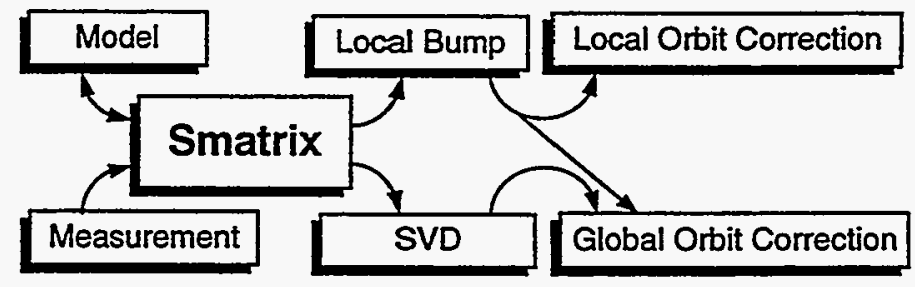

Fig.1. Smatrix-based orbit control.

This scheme does not exclude the use of a model, since one can calculate and analyze Smatrices with a model. A model-based control is indispensable when insertion devices and their compensation change the lattice configuration considerably.

Going through the process of Smatrix-based orbit control helps to calibrate the model parameters at the early stage of machine operation. The most primitive, yet important, calibration are the calculation of the BPM read-out values and the determination of the transfer functions of the steering magnets done by comparing the measured and calculated Smatrices on an assumed model. The transfer function of a

- This work was supported by the Director, Office of Energy Research, Office of Basic Energy Sciences, Material Sciences Division of the U. S. Department of Energy under Contract No. DE-AC03-76SF00098.

** Electrotechnical Laboratory, Tsukuba-shi, Ibaraki 305, Japan. 
steering magnet is a current-to-field factor that can be influenced by other magnets in its neighborhood, if the magnets are close together. Although, this evaluation task is numerically simple, it does not fix the model qualitatively. For example, it is a separate task to determine the effective edge angles of a bending magnet.

\section{LOCAL ORBIT CORRECTION}

An Smatrix contains the complete information to construct a local bump. Even if a model can calculate local bumps directly, it is a good idea to calculate an Smatrix first, then transfer it to the routine of building bumps. By creating an orbit correction algorithm, not by using a model directly, but using an Smatrix as shown in Figure 2, we can simulate the algorithm very accurately. This is because the same Smatrix is used to calculate both the changes in steering magnet settings and the expected read-out values of the BPMs.

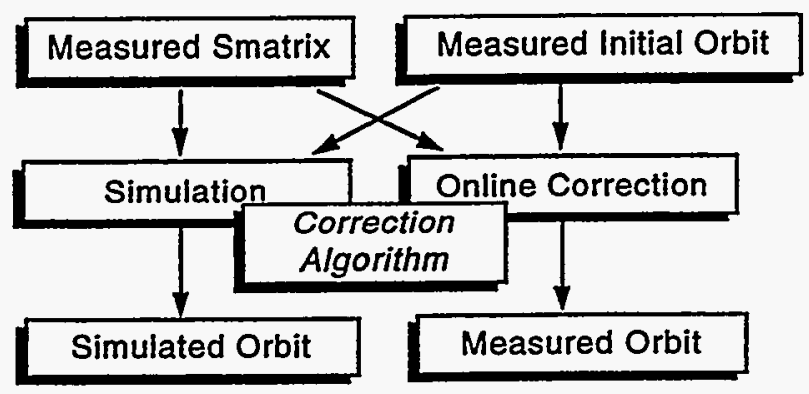

Figure 2. Measurement and simulation

This approach works very well with local orbit corrections using local bumps. Since the calibration factors are common to the measurement and the application of the Smatrix the local bumps derived from it are very precise.

At the ALS, we use local bumps to fine-adjust the orbit at the light source-points, on top of the globally corrected orbit. There are a pair of horizontal and vertical local bumps for the bending beam line to control its angles, and two pairs for each undulator beam line to control its angles and positions. This activity is supported by the program IDbump[6] implemented on a PC running Windows.

For local bumps, that are not too short, the accuracy has been enough for daily machine operation. However, short bumps with large orbit excursion require very accurately measured Smatrices.

Smatrices are also used to compensate for residual dipole kicks caused by insertion devices[7]. This is also a kind of local orbit correction.

\section{GLOBAL ORBIT CORRECTION}

We use two kinds of global orbit correction schemes: an overlapped local-bump (OLB) method and an SVD method. Both schemes were started on SUN workstations in the commissioning period[8] and we recently started using these schemes on PCs. In this paper, we focus on the recent developments in $\mathrm{C}_{++}+$on PCs running Windows NT 3.5.

We have carried out an on-line orbit correction and an off-line simulation for both schemes. Inputs are the measured initial orbit and the measured Smatrix. Outputs are the simulated orbit and the actual, measured orbit, as shown in Figure 2.

\section{A. OLB Method}

The OLB method creates local bumps using 3 correctors around the ring in a magnet pattern 1-3-5, followed by the pattern 2-4-6 and so forth. This is to avoid short bumps that require strong kicks.

\section{B. SVD Method}

The SVD method is another popular method widely used for global orbit correction, and the subroutine package is readily available[9]. The selection of the number of eigenvalues requires caution. Using a large number of the eigenvalues can improve the orbit but the corrector strengths can be too strong for practical use.

\section{Result}

The simulations and the calculations were carried out using all 96 BPMs and all correctors: 94 horizontal and 70 vertical ones. The results of simulation and actual on-line orbit correction are summarized in Table 1.

\begin{tabular}{ccccc}
\hline & $x$-rms & $y$-rms & $x$-max & $y$-max \\
\hline Initial & 0.544 & 0.545 & 1.88 & 1.74 \\
OLB sim. & 0.227 & 0.189 & 0.866 & 0.964 \\
OLB exp. & 0.225 & 0.188 & 0.920 & 0.971 \\
SVD sim. & 0.381 & 0.189 & 1.44 & 0.975 \\
SVD exp. & 0.383 & 0.218 & 1.54 & 1.06 \\
\hline
\end{tabular}

Table 1. Residual orbits [mm] after global orbit corrections

\section{Discussion}

OLB Method A model calculation shows that the residual closed-orbit distortion (COD) is estimated to be under 200 $[\mu \mathrm{m}]$. The results of the actual on-line corrections and off-line simulations are given in Table 1 . The maximum kick angle was limited to $1.0 \mathrm{mrad}$ horizontally and $0.5 \mathrm{mrad}$ vertically. OLB was carried out in only 3 iterations around the ring because it saturates in further iteration steps, maintaining the rms error and slowly lowering the maximum value. This is considered to be the effect of the lengthening of the closedorbit path. This lengthening effect is currently being investigated. 
The agreement between the measured orbit and the simulation is extremely good, as shown in Figure 3 . The maximum error is less than $100 \mu \mathrm{m}$ horizontally and $50 \mu \mathrm{m}$ vertically. Average deviations are $30 \mu \mathrm{m}$ and $20 \mu \mathrm{m}$ in both planes respectively.

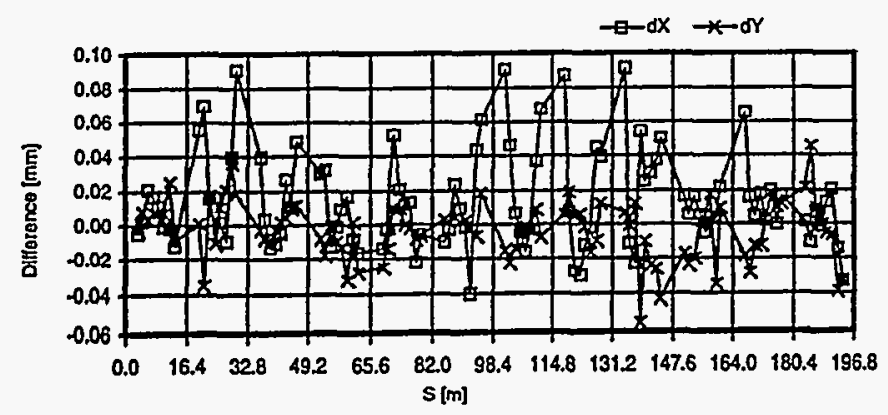

Figure 3. Difference between measurement and simulation with the OLB method

SVD Method The values in Table 1 obtained with the SVD methods are the result of using 40 eigenvalues and 3 iterations. Figure 4 shows the difference between the measured orbit and the simulation. Average deviations are 30 $\mu \mathrm{m}$ and $120 \mu \mathrm{m}$ in both planes respectively. In the vertical plane this difference is six times larger than the one obtained with the OLB method. The reason for this large difference comes from the fact that the SVD method makes use of the whole S-matrix to correct the orbit. But, the agreements between simulation and measured orbit in rms values and maximum displacements are good.

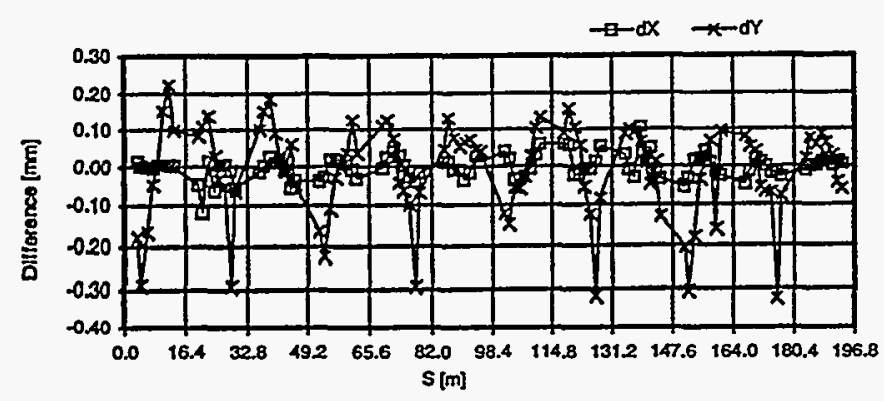

Figure 4. Difference between measurement and simulation with the SVD method

\section{DISCUSSION}

An Smatrix-based orbit control is model-free and works accurately for a fixed lattice configuration. The off-line simulation can be done very accurately. It is complementary to a model-based orbit control and crucial in the early phases of operation. The technique also serves to calibrate the model used for a model-based orbit control which is required the operation mode when lattice configurations change.

\section{ACKNOWLEDGMENTS}

The authors thank all the members of the ALS Accelerator Group and of the Controls Section, especially D. Robin and G. Portmann, for technical discussions. We also thank C. Timossi and M. Valdez for their software support, and the ALS Operators for their help in carrying out machine studies. We particularly appreciate the helpful advice from A. Jackson, R. Keller and K. Van Dongen.

\section{REFERENCES}

[1] "1-2 GeV Synchrotron Radiation Source, Conceptual Design Report," LBL PUB-5172 Rev. LBL,1986. A. Jackson, "Commissioning and Performance of the Advanced Light Source", IEEE 93PAC, 93CH3279-7, 1432, 1993.

[2] P. Kuske, LSAP-137, LBL Internal Report, 1993

[3] J. Safranek, M.J. Lee, SLAC-PUB-6442, Feb.

[4] L. Schachinger, LSAP-188, LBL Internal Report, 1994.

H. Nishimura, LSAP-185, LBL Internal Report, 1994.

D. Robin, private communication.

[5] K. J. Kleman, "Beam Diagnostics and Control at Aladdin", Nuclear Inst. and Meth., A266, 172, 1988. Y. Chung, G. Decker, and K. Evans, Jr., J. Safranek, I. So, Y. Tang, W.J. Corbett, and R. Hettel, "Global DC Closed Orbit Correction Experiment on the NSLS X-ray Ring and SPEAR", IEEE PAC, p.2275, 1993.

[6] H. Nishimura, LSAP-182, LBL Internal Report, 1994.

[7] G. Portmann and C. Timossi, LSEE-188, LBL Internal Report, 1994

[8] L. Schachinger, LSAP-183, LBL Internal Report, 1994.

[9] W.H. Press, B.P. Flannery, S.A. Teukolsky, W.T.

Vetterling, Numerical Recipes in C, Cambridge University Press, P60, 1989. 